\title{
Aspectos jurídicos de la custodia de documentos electrónicos
}

Legal aspects of the custody of electronic documents

\author{
José Félix MuÑoz SORO \\ Agencia Aragonesa para la Investigación y el Desarrollo (ARAID), \\ Edificio I+D, Parque Tecnológico Walqa, 22917 Cuarte (Huesca), jfm@unizar.es
}

\begin{abstract}
Resumen
La naturaleza jurídica de la custodia documental cambia al pasar de realizarse con documentos en soporte papel a hacerlo con documentos electrónicos. Con la regulación actual, deja de constituir un contrato de depósito, aunque siguen resultando aplicables muchas de las obligaciones del depositario. Sí que puede considerarse como un servicio de la sociedad de la información, por lo que es aplicable lo establecido para estos en la Ley 34/2002, de 11 de julio, de Servicios de la Sociedad de la Información, y, cuando los documentos contengan datos personales, también como un tratamiento de datos por cuenta de terceros. Pero, los cambios más importantes se deben a la constitución, por parte del Reglamento eIDAS, de la preservación de firmas y sellos electrónicos como un servicio de confianza. La aparición de esta nueva figura plantea numerosos interrogantes sobre quiénes son los agentes indicados para realizar cada una de las funciones asociadas a la conservación de los documentos electrónicos auténticos durante periodos prolongados de tiempo.
\end{abstract}

Palabras clave: Documentos electrónicos. Custodia. Servicios de confianza. Reglamento elDAS. Firma electrónica longeva. Unión Europea. España.

\section{Introducción}

En las últimas décadas se ha generalizado en las empresas y, más recientemente, también en las Administraciones públicas, la externalización (outsourcing) de determinadas funciones, por motivos de eficacia y economía. Una de las áreas que suele externalizarse son los sistemas administrativos $\mathrm{y}$, dentro de esta, las tareas relativas al archivo de documentos.

Entre los motivos para ello se encuentran los inconvenientes que plantea la llevanza y custodia del archivo físico, y los costes que conlleva el que este se ubique en el mismo lugar que las oficinas de la empresa o Administración, situadas normalmente en zonas donde el espacio suele tener un precio elevado. Por tanto, una primera necesidad de los clientes es que se custodie su archivo de forma apropiada, pero fuera de su sede. A este servicio de mera con-

\begin{abstract}
The legal nature of document custody changes when it moves from paper documents to electronic ones. With the current Spanish regulation, it no longer constitutes a deposit agreement, although many of the depositary's obligations continue to be applicable. On the other hand, it can be considered as a service of the information society and therefore is applicable the liability regime established for them in the Spanish Law of Services for the Information Society, and also as a processing of data on behalf of third party, when documents contain personal data. However, the most important changes are due to the constitution by the eIDAS European Union Regulation of the preservation of electronic signatures and electronic stamps as a trust service. The emergence of this new figure raises numerous questions about who are the agents indicated to carry out each of the functions associated with the preservation of authentic electronic records for extended periods of time.
\end{abstract}

Keywords: Electronic documents. Archiving. Trust services. Regulation (EU) elDAS. Long-Term digital signature. European Union. Spain.

servación habrán de añadirse, como mínimo, los necesarios para poder recuperar cualquiera de los documentos archivados cuando así se solicite. $Y$ también suelen añadirse servicios de transporte, como son la recogida en las sedes de los clientes de los documentos que van a ser archivados y la entrega en las mismas de los documentos cuya recuperación se solicita. No hay una regulación específica para este conjunto de prestaciones que forman el servicio de custodia documental, por lo que se trata de un contrato atípico y también, desde el punto de vista de la ley, innominado.

Con la utilización creciente de los documentos electrónicos surge la necesidad de su archivo y custodia. Ahora los motivos no tienen nada que ver con la ocupación del espacio físico. De hecho, la mera conservación de los archivos formados por ficheros informáticos- parece no necesitar de agentes especializados, ya que el 
auge de la computación en la nube ha hecho habitual el servicio de almacenamiento de contenidos, del que son ejemplos muy conocidos Dropbox, Drive o iCloud. Pero, el servicio de custodia de documentos electrónicos no solo incluye la aplicación de las técnicas de gestión documental necesarias para garantizar su localización, como ya se ha dicho, sino que ahora se hacen precisas nuevas prestaciones, ya que los documentos electrónicos auténticos necesitan de la adopción de ciertas medidas y de la realización de determinadas operaciones para mantener su fuerza probatoria a lo largo del tiempo.

En el artículo se analiza la naturaleza jurídica del servicio de custodia de documentos electrónicos, teniendo en cuenta las distintas figuras jurídicas que concurren en el mismo. La primera es el contrato de depósito (§2), dentro del que se encuadra la custodia de documentos en papel, pero, difícilmente, la de documentos electrónicos, que, sin embargo, sí puede ser considerada como un servicio de la sociedad de la información (§3). En ambos casos, si los documentos contienen datos de carácter personal, se produce un tratamiento de datos por cuenta de tercero, regulado en la normativa de protección de datos (\$4). Por último, si la custodia incluye documentos electrónicos auténticos, es preciso dilucidar si constituye en sí misma un servicio de confianza o si es suficiente con que el custodio haga uso de estos servicios para la realización de determinadas operaciones (§5).

\section{Como contrato de depósito}

\subsection{Depósito de documentos en soporte papel}

La definición del depósito viene dada por el artículo 1758 del Código Civil, según el cual "se constituye el depósito desde que uno recibe la cosa ajena con la obligación de guardarla y de restituirla". El ámbito en el que el deposito es más utilizado es el mercantil, en el que, entre otros muchos supuestos, existen entidades especializadas en el depósito de mercaderías como son los Almacenes Generales de Depósitos- o de efectivo, ya que el deposito es la base de las operaciones de captación de pasivo por parte de las entidades de crédito (Uria et al., 2001, p. 297).

Respecto a la naturaleza, civil o mercantil, del contrato de custodia, el artículo 303 del Código de Comercio establece que "para que el depósito sea mercantil se requiere: $1^{\circ}$ ) Que el depositario, al menos, sea comerciante. $2^{\circ}$ ) Que las cosas depositadas sean objeto de comercio. $3^{\circ}$ ) Que el depósito constituya por sí una operación mercantil, o se haga como causa o a conse- cuencia de operaciones mercantiles". Pero, para calificar un deposito como mercantil no es necesaria la concurrencia de los tres requisitos, siendo suficiente que el depositario sea comerciante y que el deposito se realice en virtud de un acto de comercio, condiciones que se cumplen en las empresas de custodia documental.

En cuanto a la perfección del contrato, dado que el artículo 305 del Código de Comercio dispone que "el depósito quedará constituido mediante la entrega al depositario de la cosa que constituya su objeto", la doctrina tradicionalmente ha considerado al depósito como un contrato real, que únicamente se perfecciona con la entrega del objeto depositado (Uria et al., 2001, p. 298). Sin embargo, otros autores opinan que el contrato puede perfeccionarse, como cualquier otro, mediante el consentimiento de las partes, y que el momento de la entrega simplemente determinará el inicio de las obligaciones de custodia y devolución del depositario (Broseta et al., 2010, p. 147). Esta segunda interpretación es, a nuestro juicio, más acorde con la práctica de empresas como las de custodia documental, ya que en estas el contrato se perfecciona cuando el cliente acepta la prestación de los servicios, originándose desde este momento obligaciones como, por ejemplo, el posible pago de una cuota periódica por parte del depositante o la puesta a disposición del servicio de recogida por parte del depositario.

De hecho, las propuestas normativas y académicas más recientes consideran al depósito como un contrato consensual (Sirvent, 2015, p. 30). Así ocurre en el Anteproyecto de ley del Código Mercantil —aprobado por el Gobierno en 2014 y aun en tramitación- cuyo artículo 551 dice que "por el contrato de depósito se obliga, a cambio de un precio, a custodiar las cosas que le entregue el depositante y a devolvérselas". En la misma línea, el Proyecto de un marco común de referencia para el derecho privado europeo (Draft Common Frame of Reference DCFR), una iniciativa académica que pretende servir de base para la elaboración de un futuro derecho contractual europeo (Jerez Delgado, 2015) también considera al depósito como un contrato de prestación de servicios de carácter consensual.

\section{2. ¿Depósito de documentos electrónicos?}

Según el artículo 1761 del Código Civil una de las características inherentes al contrato de depósito es que su objeto sean cosas muebles. Los documentos en soporte papel lo son, de forma que no plantea problemas considerar a la custodia de estos documentos como un contrato 
de depósito. Por suparte, os documentos electrónicos, según el Reglamento (UE) 910/2014, del Parlamento Europeo y del Consejo, de 23 de julio de 2014, relativo a la identificación electrónica y los servicios de confianza para las transacciones electrónicas en el mercado interior y por el que se deroga la Directiva 1999/93/CE (Reglamento elDAS), son "todo contenido almacenado en formato electrónico, en particular, texto o registro sonoro, visual o audiovisual". El almacenamiento en formato electrónico cambia de forma radical la naturaleza del documento, que pasa de ser una cosa mueble a convertirse en un conjunto de datos normalmente una secuencia binaria- cuya existencia e identidad como documento es independiente del soporte o soportes en los que estén registrados. Por tanto, también cambia la naturaleza del contrato de custodia, ya que se pasa de almacenar objetos materiales a conservar entidades inmateriales.

No debe llevarnos a confusión el hecho de que en ocasiones la doctrina denomine depósito a contratos cuyo objeto es, en puridad, un documento electrónico. Un ejemplo es el escrow de programas informáticos que Soler (2016, p. 983) denomina "contrato de depósito de código fuente". Con carácter general, el término escrow se refiere al establecimiento de una garantía sobre el cumplimiento de un contrato basada en el depósito de un bien en manos de un tercero de confianza (STS 4811/2014). En el caso de los programas de ordenador se deposita el código fuente, de forma que, por una parte, el desarrollador del programa mantiene el secreto del mismo y, por otra, el adquirente tiene la garantía de que, en determinadas circunstancias, podrá acceder a dicho código, lo que le permitirá realizar el mantenimiento y actualización de los programas. Pero, en este caso, lo que realmente constituye el objeto del depósito es el soporte físico que almacena la copia del código fuente.

La custodia de documentos electrónicos no se refiere ni a copias únicas, ni a los soportes que las contienen - de hecho, cabe prever que la operatoria más frecuente para su entrega será la remisión a través de las redes telemáticas, de forma que el depositario los recibirá a través un flujo binario- sino a documentos que, aunque estarán siempre almacenados en uno $u$ otro soporte, son en sí mismos entidades inmateriales. Ello supondría, según Bercovitz (2011, p. 318) la imposibilidad de aplicar la figura del contrato de depósito ya que en este "parece lógico que habrá de tratarse de cosas susceptibles de entrega y traslado posesorio, esto es, dotadas de naturaleza material o corporal, lo que seguramente excluye el depósito de dere- chos que no estén incorporados a un título, así como el depósito de bienes inmateriales".

Pero, aunque dicha posición se justifique a tenor de la regulación actual del contrato de depósito, la consideración de este como un contrato de servicios en el contexto del tráfico económico del siglo $X X I$, en el que cada vez juegan un papel más importante entidades inmateriales como los datos o los documentos electrónicos, hace necesario superar esta limitación y extender el ámbito objetivo del depósito. Así se hace en el mencionado Proyecto de un marco común de referencia para el derecho privado europeo, que incluye en el objeto del contrato de depósito tanto a los bienes muebles como a las cosas incorporales (incorporeal things).

\subsection{Obligaciones del depositario}

Se considere o no al servicio de custodia de documentos electrónicos como un contrato de depósito, hay elementos del mismo que, aunque sea por analogía, podrían considerarse aplicables, en especial, algunas de las obligaciones que la ley establece para las partes del mismo $y$, sobre todo, para el depositario. Estas obligaciones tienen su origen en la exigencia de una actitud activa del depositario, el cual no cumple con una mera custodia de la cosa depositada, sino que debe realizar todas las acciones precisas para garantizar que esta no sufra ningún perjuicio o menoscabo. Precisamente de esta obligación deriva el carácter oneroso del contrato de depósito, ya que se exige al depositario que despliegue un esfuerzo suficiente y de forma continuada.

En el caso de los documentos electrónicos la defensa de la cosa depositada no es, ni mucho menos, más sencilla que en los documentos en soporte papel, ya que les amenazan numerosas vulnerabilidades y riesgos. En primer lugar, se encuentran los derivados de su naturaleza volátil, ya que un minino daño en el soporte físico que los conserva o la degradación del mismo puede llevar fácilmente a la perdida de los documentos. La medida de seguridad básica frente a este riesgo es la redundancia, que exige conservar varias copias, en distintos sistemas y lugares diversos. La pérdida de la cosa depositada lleva al incumplimiento de la obligación básica del depositario, que es su devolución o, en el caso de los documentos electrónicos, la puesta a disposición.

Pero el depositario no responde solo por la pérdida de los documentos, sino también por cualquier menoscabo que puedan sufrir estos. Así, por ejemplo, el artículo 308 del Código del Comercio dispone que el depositario de "títulos, 
valores, efectos o documentos que devenguen intereses", tendrá la obligación de "practicar cuantos actos sean necesarios para que los efectos depositados conserven el valor y los derechos que les correspondan con arreglo a las disposiciones legales". Análogamente, el depositario de documentos electrónicos auténticos deberá realizar todas las acciones precisas para que estos no pierdan los efectos jurídicos derivados de su autenticidad. Obligación que, como se verá en el epígrafe 5 , es uno de los aspectos fundamentales de la custodia de estos documentos.

Distinto carácter tiene el riesgo de posibles accesos indebidos a los documentos custodiados, ya que este no supone propiamente un menoscabo de los mismos. Sin embargo, habrá como mínimo un incumplimiento contractual del depositario si la obligación de confidencialidad se ha incluido en el contrato del servicio de custodia. Además, pueden producirse otros perjuicios para el depositante, derivados de las obligaciones que, como titular de la información incluida en los documentos, le atribuyan la legislación sobre protección de datos de carácter personal, a la que nos referiremos más adelante, u otras normas, como las relativas al secreto comercial o al que cubre a determinada información pública. Por tanto, la obligación de diligencia del depositario se hace aquí particularmente importante y para valorar cuál debe ser su alcance puede recurrirse a las normas técnicas sobre seguridad de los sistemas de información y a la normativa sobre administración electrónica, en especial, al Real Decreto 3/2010, de 8 de enero, por el que se regula el Esquema Nacional de Seguridad en el ámbito de la Administración Electrónica.

\section{Como servicio de la sociedad de la información}

La Ley 34/2002, de 11 de julio, de Servicios de la Sociedad de la Información (LSSI) define estos servicios como aquellos que se prestan a distancia, por vía electrónica y a petición individual del destinatario, normalmente a título oneroso. Dentro de esta amplia definición cabria incluir a la custodia de documentos electrónicos, ya que tanto de entrega de los documentos por el depositante como la puesta a disposición por el depositario se producirá, típicamente, a distancia, a través de redes telemáticas, siendo el propio objeto del servicio - los documentos electrónicos - entidades de naturaleza informática. En consecuencia, les será de aplicación lo dispuesto en la LSSI como, por ejemplo, el principio de libre prestación de los servicios, que se establece en el artículo 7.
Dentro de los servicios de la sociedad de la información la LSSI establece una categoría específica que denomina servicios de intermediación. Lopez-Monis (2003: p. 39) considera que se distingue entre los servicios finales, que serían aquellos que satisfacen directamente una necesidad del usuario y los servicios de intermediación, que únicamente contribuyen a acceder a los anteriores pero que en sí mismos no satisfacen dicha necesidad. Pero, al menos en determinados supuestos, este criterio no resulta muy claro. Por ejemplo, YouTube es un servicio al que la sentencia 289/2010, de 20 septiembre, del Juzgado de lo Mercantil $\mathrm{n}^{\circ} 7$ de Madrid, considera de intermediación, basándose en que no interviene ni directa ni indirectamente en la creación de los contenidos que publica. Sin embargo, difícilmente podríamos decir que YouTube no satisface las necesidades finales de sus usuarios, tanto de aquellos que suben videos para compartir como de quienes los visualizan.

Entre los servicios de intermediación se encuentra el de alojamiento o almacenamiento de datos. El artículo 16 de la LSSI establece un régimen de responsabilidad especifico, según el cual quienes presten un servicio de alojamiento o almacenamiento no serán responsables en los supuestos de tenencia de información ilícita o que lesione derechos de terceros cuando "no tengan conocimiento efectivo de que la actividad o la información almacenada es ilícita o de que lesiona bienes o derechos de un tercero [...] o, si lo tienen, actúen con diligencia para retirar los datos o hacer imposible el acceso a ellos". Si consideramos a la custodia de documentos electrónicos, al menos por su naturaleza técnica, como un servicio de alojamiento o almacenamiento de datos, le sería aplicable esta disposición, de lo que derivarían tres consecuencias relevantes.

La primera es que sería aplicable a los prestadores del servicio de custodia documental la exención que se establece respecto a la obligatoriedad de realizar un control previo de la información que se recibe. De acuerdo con la precitada sentencia "no es posible imponer a ningún prestador de servicios de intermediación una obligación general de supervisar los datos que se transmitan o almacenen". En el caso de los servicios de custodia documental, además, habría de tenerse en cuenta que la previa supervisión de los documentos depositados, en caso de realizarse, podría entrar en conflicto con derechos fundamentales del depositante, como el secreto de las comunicaciones o la privacidad. 
La segunda consecuencia es relativa a las responsabilidades que podrían derivarse de la presencia de información ilícita o que origine daños a terceros, en los documentos depositados. Al regular los servicios de alojamiento o almacenamiento la LSSI se refiere sobre todo a aquellos casos en los que el almacenamiento de datos tiene como finalidad principal su difusión -como, por ejemplo, en los servidores web-y no la mera conservación. Dado que los posibles perjuicios causados por la información ilícita siempre serán menores cuando la información no se hace accesible a otras personas, sino que únicamente se mantiene a disposición de quien, en su momento, hizo entrega de la misma, cabe considerar que, aplicando la analogía a maiori ad minus, en el servicio de custodia de documentos electrónicos se justifica en mayor medida la exención de la responsabilidad del prestador del servicio por la presencia de este tipo de información.

La tercera consecuencia trata de la forma en que debe actuar una empresa que custodia documentos electrónicos cuando conozca su contenido ilícito. La LSSI exige que el prestador del servicio cuando sea conocedor de la presencia de información ilícita "actúe con diligencia para retirar los datos o hacer imposible el acceso a ellos", por lo que parece que basta con que se hiciera imposible el acceso — se supone que del público- a los mismos. Normalmente, esta condición se cumple en el servicio de custodia prestado por empresas, pero la disposición sí sería aplicable a los archivos cuyos documentos son accesibles a través de Internet. También cabe plantearse si a los prestadores del servicio de custodia les puede convenir establecer cláusulas contractuales que permitan la eliminación de la información ilícita o que perjudique a terceros, pero sin olvidar que hay supuestos en los que puede darse una causa justificada para la conservación de contenidos que en sí mismos son ilícitos como, por ejemplo, la custodia de expedientes judiciales o administrativos en los que se encuentren incluidos.

\section{Como tratamiento de datos de carácter personal}

La norma básica sobre protección de datos en nuestro país es la Ley Orgánica 15/1999, de 13 de diciembre, de protección de datos de carácter personal (LOPD). A partir del 25 de mayo de 2018 entrará en vigor el Reglamento (UE) 2016/679 del Parlamento Europeo y del Consejo, de 27 de abril de 2016, relativo a la protección de las personas físicas en lo que respecta al tratamiento de datos personales y a la libre circulación de estos datos y por el que se dero- ga la Directiva 95/46/CE (Reglamento general de protección de datos, RGPD). Ambas normas consideran tratamiento de datos a cualquier operación o conjunto de operaciones realizadas sobre datos personales o conjuntos de datos personales, como la recogida, registro, organización, estructuración, conservación, adaptación o modificación, extracción, consulta, etc. De la mención expresa a la conservación cabe concluir que la custodia documental, tanto de documentos en soporte papel como electrónicos, constituye tratamiento de datos siempre, claro está, que los documentos contengan datos de carácter personal. De acuerdo con los artículos 12 de la LOPD y 28 del RGPD, el responsable del tratamiento puede delegar la realización de parte de este o de la totalidad en otra persona, a la que se denomina encargado del tratamiento.

Previamente al comienzo de la actividad del encargado este y el responsable deben realizar un contrato, del que habrá de quedar constancia y para cuyo contenido la Comisión y las autoridades de control en materia de protección de datos podrán fijar cláusulas contractuales tipo. En el contrato se recogerán necesariamente, entre otras, la obligación del encargado de tratar los datos personales siguiendo las instrucciones documentadas del responsable, su compromiso de tratamiento confidencial, de adoptar las medidas de seguridad según el RGPD, y de asistir al responsable para que pueda cumplir con su obligación de responder a las solicitudes de los interesados para el ejercicio de los derechos ARCO (acceso, rectificación, cancelación y oposición). El prestador del servicio de custodia documental, como encargado del tratamiento, no puede utilizar los datos con fines distintos a los previstos en el contrato, y no puede recurrir a otro encargado - por ejemplo, mediante subcontratación- "sin la autorización previa por escrito, específica o general, del responsable". En este caso, al nuevo encargado deberán imponérsele las mismas obligaciones que tiene el primero. Una vez finalizada la prestación del servicio el encargado del tratamiento no puede conservar los datos, debiendo, a elección del responsable del tratamiento, devolver, destruir o borrar de forma segura los soportes utilizados. $\mathrm{Si}$ el encargado incumple estas obligaciones, será considerado también responsable del tratamiento y responderá como tal.

En cuanto a las medidas de seguridad, el RGPD aplica el principio de proporcionalidad al establecer que la implementación de unas u otras se hará en función del resultado de una valoración del riesgo que se realizará para cada caso, atendiendo no solo al tipo de datos, como ocu- 
rre en la regulación actual, sino también al entorno en el que se realiza el tratamiento. Las obligaciones de seguridad derivadas de la normativa de protección de datos, aunque se refieren únicamente a los documentos que contengan datos personales, concuerdan plenamente con las que son propias de la diligencia debida por el depositario en la conservación de la cosa depositada. Por ello, pueden tomarse el RGPD y su normativa de desarrollo como referencia para la aplicación de las medidas de seguridad a todos los documentos custodiados, sin perjuicio de que haya determinados conjuntos de documentos que precisen de niveles superiores de seguridad, por ejemplo, por contener datos especialmente protegidos.

\section{Como servicio de confianza}

\subsection{El servicio de conservación de firmas y sellos electrónicos}

Aunque hasta aquí el presente artículo se ha centrado en los aspectos jurídicos de la custodia de documentos electrónicos realizada por empresas dedicadas a esta actividad, los aspectos del Reglamento eIDAS que se van a estudiar en este epígrafe afectan en igual forma a todos los archivos de documentos electrónicos auténticos, tanto del sector público como del privado.

El Reglamento eIDAS regula la prestación de servicios de confianza en relación con los documentos electrónicos, siempre que los efectos jurídicos de los documentos no se limiten al ámbito interno de una organización. A los servicios previamente regulados -la emisión de certificados de clave pública, la emisión de sellos de tiempo y la validación de firmas y sellos electrónicos - añade otros dos. Uno es el de entrega electrónica certificada, que equivale a la notificación electrónica, de uso ya muy extendido en la administración electrónica. El segundo es la preservación de firmas, sellos o certificados electrónicos relativos a los anteriores servicios (artículo 3, 16, c). El artículo 34 regula este servicio, al que denomina de conservación, cuando se presta sobre firmas electrónicas y el 40 extiende la misma regulación a los sellos electrónicos.

Existen dos motivos fundamentales que hacen que las firmas electrónicas avanzadas y los sellos electrónicos avanzados pierdan su validez con el transcurso del tiempo. Uno de ellos es la caducidad de los certificados que forman la cadena de confianza que los respalda y que permite su validación. Esta se produce en plazos de tiempo prefijados, ya que todos los certificados deben indicar su periodo de validez. El segundo es la debilitación de la garantía que ofrecen los algoritmos criptográficos utilizados, por haber evolucionado la técnica hasta el punto de hacerlos demasiado vulnerables para su aplicación. Por tanto, la conservación de documentos electrónicos auténticos no puede limitarse a una mera custodia pasiva, sino que para mantener su validez a lo largo de periodos extensos de tiempo es precisa la realización de determinadas operaciones y el cumplimiento de exigentes requerimientos técnicos. Entre estos es particularmente importante la utilización de firmas o sellos longevos, en los que se integran en un fichero los elementos (certificados, sellos de tiempo y respuestas OCSP) que permiten verificar la autenticidad, junto a la propia firma o sello, utilizando un formato estándar. Previamente a la caducidad de los certificados que contiene, se aplica sobre la firma un sello y con ello su validez se prolonga durante el periodo de vigencia que tenga el sello utilizado para el resellado (Muñoz et al., 2014, p. 53).

De la redacción del ya mencionado artículo 34 del Reglamento elDAS, según la cual "solo podrá prestar un servicio cualificado de conservación de firmas electrónicas cualificadas el prestador cualificado de servicios de confianza que utilice procedimientos y tecnologías capaces de ampliar la fiabilidad de los datos de la firma electrónica cualificada más allá del período de validez tecnológico", se desprende claramente que esta capacidad de prolongar en el tiempo la validez de las firmas y sellos constituye el núcleo del servicio de conservación.

La prestación de los servicios de confianza es libre, pero para que un servicio tenga la consideración de cualificado es preciso cumplir los requisitos que establece el artículo 24 del $\mathrm{Re}$ glamento elDAS. Esta circunstancia debe quedar acreditada mediante un informe de evaluación de la conformidad (CAR) expedido por un organismo de evaluación debidamente acreditado. Luego, la puesta en marcha del servicio ha de notificarse al organismo de supervisión, que en España es el Ministerio de Energía, Turismo y Agenda Digital, el cual, si todo es conforme, lo incluirá en la lista de confianza, regulada en el artículo 22, momento a partir del cual podrá comenzar la prestación del servicio de confianza cualificado.

La Guía de notificación de servicios electrónicos del mencionado Ministerio (2016) indica, en primer lugar, la conveniencia de tener en cuenta los estándares técnicos definidos por el European Telecommunications Standard Institute (ETSI), de los que será de aplicación en todo caso la norma ETSI 319401 General Policy Requirements for Trust Service Providers. Lue- 
go exige que todos los servicios cualificados dispongan de una Declaración de prácticas, de un Plan de cese, y de los modelos de contratos empleados para formalizar la prestación de sus servicios de confianza con los clientes, así como en la relación con proveedores y entidades en las que delegue todo o parte de su actividad. A ello se añade la obligatoriedad de disponer de un seguro de responsabilidad civil o de un aval bancario o seguro de caución por un importe de al menos 3 millones de euros. En el caso del servicio de conservación de firmas y sellos electrónicos se deberá disponer además de la correspondiente Política de conservación y de un certificado de resellado. Como consecuencia de todo ello la constitución de un servicio cualificado de confianza resulta bastante onerosa.

\subsection{La desagregación de los documentos y las evidencias}

De acuerdo con su definición del servicio de conservación cabe deducir que para el Reglamento elDAS el objeto del mismo son únicamente las firmas y sellos electrónicos y, en ningún caso, los documentos electrónicos (Dumortier, 2016, p. 13). En este sentido, parece que se le da un cierto carácter auxiliar respecto a los demás servicios de confianza y se limita su ámbito a las evidencias, sin hacer ninguna mención a los documentos autentificados. Esta situación no es nueva, ya que desde los primeros periodos de la regulación y uso de la firma electrónica esta última ha sido tratada como si fuera una entidad independiente, casi ajena a los documentos electrónicos, ignorando con ello que, al igual que ocurre con los documentos en soporte papel, la firma no es sino un instrumento de autentificación que solo cobra sentido incorporada a un documento. Puede que a ello haya contribuido el hecho de que el mismo medio - los certificados - sea utilizado tanto para la identificación de las personas como para la autentificación de los documentos, doble función que ha dado lugar con frecuencia a regulaciones confusas. Pero esta distinción no está presente en el caso del archivo, que habrá de practicarse siempre sobre documentos, ya que la identificación es un acto que se consume en el momento mismo en que se realiza. Por tanto, las firmas y sellos que se preserven estarán vinculados a un documento autentico y la custodia de documentos electrónicos auténticos será siempre, al menos en parte, un servicio de confianza.

Por otra parte, debe tenerse en cuenta que la problemática asociada a la preservación de los documentos auténticos a lo largo de periodos prolongados de tiempo no se limita a los aspec- tos ya mencionados -caducidad de los certificados y obsolescencia de los algoritmos criptográficos- sino que también tiene que ver con la legibilidad de los documentos. Esta circunstancia se refleja, por ejemplo, en el artículo 17.2 de la Ley 39/2015, de 1 de octubre, del procedimiento administrativo común de las administraciones públicas, que establece que los documentos electrónicos deberán conservarse en un formato que, con independencia del tiempo transcurrido desde su emisión, permita garantizar no solo la autenticidad e integridad, sino también su consulta.

Para cumplir con esta obligación de mantener la legibilidad, la posible obsolescencia o la inadecuación para el archivo de los formatos utilizados cuando se creó un documento o en fases anteriores del archivo pueden obligar a la conversión a otro formato más reciente o apropiado, lo que inevitablemente afecta a la validez de las firmas y sellos y exige la generación de nuevos medios de autentificación, operación esta última que sería propia de la prestación de servicios de confianza. Además, el mencionado artículo 17.2 dispone que "se asegurará en todo caso la posibilidad de trasladar los datos a otros formatos y soportes que garanticen el acceso desde diferentes aplicaciones". El traslado a otros formatos, cuando el documento haya de conservar su carácter de documento autentico, exigirá también de la realización de operaciones de firma o sellado por parte del responsable de la custodia.

La conservación de los documentos en papel viene siendo realizada por los archivos, órganos especializados en esta función, la cual realizan íntegramente. Su número es elevado y su tipología muy diversa, ya que muchas organizaciones, tanto públicas como privadas, disponen del mismo o recurren a las empresas de custodia, y están también aquellos cuya función es la conservación del patrimonio histórico documental. Por el contrario, el numero de prestadores cualificados de servicios de confianza es bastante pequeño e incluye algunas instituciones FNMT, Cámaras de Comercio, Notarios, Registradores- y unas pocas empresas. Dada la dificultad y el coste que supone la creación de un servicio cualificado de confianza, cabe suponer que serán estas entidades las que se constituyan como prestadores de servicios cualificados de conservación de firmas y sellos electrónicos.

Podría parecer que la regulación conduce a un escenario en el que los documentos serán conservados por unos agentes y las evidencias que los autentifican por otros distintos. Pero no tiene por qué ser así, ya que es posible que los archivos sigan cumpliendo con su función tradicional, 
conservando la totalidad del documento, y que el servicio cualificado de conservación se limite al resellado de firmas longevas, operacion que realizaría cuando fuera requerido para ello por el custodio de los documentos. La cuestión que habrá de dilucidarse es si el servicio regulado por el Reglamento elDAS incluye necesariamente la custodia de las evidencias por quien lo presta, como parece sugerir el termino conservación utilizado por la norma, o si puede limitarse a la realización de las operaciones de resellado, como cabe deducir del tenor literal del artículo 34, que únicamente se refiere a la función de "ampliar la fiabilidad de los datos de la firma electrónica cualificada más allá del período de validez tecnológico".

\subsection{La fuerza probatoria de las firmas longevas}

La piedra angular de la regulación sobre firma electrónica son el artículo 25.2 del Reglamento eIDAS, según el cual "una firma electrónica cualificada tendrá un efecto jurídico equivalente al de una firma manuscrita", y el 35.2 que dispone que "un sello electrónico cualificado disfrutará de la presunción de integridad de los datos y de la corrección del origen de los datos a los que el sello electrónico cualificado esté vinculado". Estas disposiciones hacen que los documentos electrónicos autentificados con una firma o sello cualificados puedan cumplir las mismas funciones que sus homólogos en soporte papel.

La cuestión es si para que una firma o sello cualificados conserven esta cualidad tras un proceso de resellado, este deberá ser realizado por un prestador cualificado del servicio cualificado de conservación. Como se vio, el artículo 34 del Reglamento elDAS, dispone que solo podrá prestar un servicio cualificado de conservación de firmas electrónicas cualificadas un prestador cualificado de servicios de confianza que disponga de medios para ampliar la fiabilidad de los datos de la firma electrónica cualificada más allá del período de validez tecnológico, pero no dice expresamente que solo tendrán está capacidad dichos prestadores. Sin embargo, de no ser así, cualquiera que dispusiera de una firma o sello cualificado podría resellar firmas y sellos cualificados sin que estos perdieran fuerza legal y, en consecuencia, carecería de sentido la creación del servicio cualificado de conservación.

En nuestro país, la utilización de documentos electrónicos auténticos ha comenzado sobre todo en las Administraciones públicas y es también en este contexto donde se desarrollan las primeras iniciativas de archivo electrónico, entre las que cabe destacar el proyecto Archive. En estas experiencias pioneras la práctica que se suele seguir para el resellado de firmas longevas es utilizar un sello cualificado de la propia Administración que emitió en su día el documento, de forma que la operación está siendo realizada por una entidad que no es un prestador cualificado de servicios de confianza. Podría argumentarse que al ser la propia organización que autentificó en su día un documento con su sello la que lo resella de nuevo, este nuevo sello cualificado substituye de alguna forma al anterior y podría aplicarse la presunción del artículo 35.2. Pero ello supondría confundir la operación de firmar o sellar un documento con la meramente instrumental del resellado para prolongar la vigencia de una firma o sello preexistente, cuando la naturaleza y efectos de ambas operaciones son totalmente distintos. Además, el argumento no es aplicable a aquellos documentos que no se autentificaron con un sello de la Administración, sino que, en su día, fueron firmados por un empleado público con su firma electrónica cualificada. En estos aún resulta más evidente que el resellado mediante un sello de la Administración, que no es prestador cualificado del servicio de conservación de firma y sellos electrónicos, no permitiría conservar a la firma el carácter de cualificada y, con ello, dejaría de tener un valor equivalente a la firma manual.

Las consecuencias prácticas de esta interpretación son importantes y suponen, al menos en un primer momento, una nueva dificultad en el desarrollo de los archivos de documentos electrónicos. Sin embargo, como ya se ha dicho, puede cumplirse con lo dispuesto en el Reglamento eIDAS convirtiendo al resellado en un servicio que se solicite a los prestadores, de forma similar a como se hace ahora con los sellos de tiempo o con el servicio de validación, mediante respondedores OCSP. Esta forma de operar tendría la ventaja de que liberaría a los responsables de los archivos de la gestión de las firmas longevas y los sellos de resellado, así como de la realización de las operaciones criptográficas. Entre los inconvenientes se encontraría el gran volumen de documentos que suele manejarse en los archivos de las Administraciones, que podría hacer muy costosa la utilización de un recurso externo para el resellado.

El alcance que se dé al servicio cualificado de conservación de firmas y sellos desde el punto de vista jurídico, también tendrá importantes consecuencias ya que, si se considera que este incluye necesariamente la custodia de las firmas y sellos, sería necesario obtener siempre las evidencias de los repositorios gestionados por el 
prestador cualificado del servicio, lo que incrementaría aún más la complejidad técnica para la gestión y conservación de las firmas longevas de los documentos auténticos conservados en los archivos. Tanto es así que este podría considerarse como uno de los argumentos principales para desechar esta interpretación del Reglamento eIDAS.

\section{Conclusión}

Desde una óptica jurídica, dos son las transformaciones más relevantes de la custodia de documentos cuando esta pasa a realizarse sobre documentos electrónicos. La primera es que su objeto deja de ser material y se convierte en una entidad inmaterial. Se produce con ello un cambio similar al que se ha dado en otros contratos sobre contenidos - como, por ejemplo, la adquisición de obras audiovisuales a través de Internet- en el que se pasa de una regulación fundada en la propiedad y la posesión a otra basada en derechos que se adquieren frente a empresas proveedoras de servicios de la sociedad de la información. Con ello, la custodia de documentos electrónicos se aleja de la figura del depósito, al menos en la forma en la que actualmente se regula en los códigos civil y mercantil, y pasa a integrase entre los servicios de la sociedad de la información.

La segunda transformación tiene que ver con la perdurabilidad de los mecanismos técnicos utilizados para garantizar la autenticidad de los documentos electrónicos, ya que, dado que estos tienen periodos breves de obsolescencia, es preciso que el custodio realice determinadas operaciones de firma para mantener su validez a lo largo del tiempo; y, para ello, el Reglamento eIDAS ha introducido un nuevo servicio de conservación de firmas y sellos electrónicos. La existencia de proveedores cualificados de este servicio puede suponer una garantía de que las operaciones necesarias para mantener la vigencia de los documentos auténticos a lo largo del tiempo se realizan de forma correcta, evitando posibles pérdidas, no de información, pero sí de la fuerza probatoria de documentos que pueden ser importantes. Además, se dota a estos mecanismos de las garantías de fiabilidad y transparencia que deben tener todos los servicios cualificados de confianza. Y otra consecuencia que cabe esperar de la asignación de estas funciones a agentes especializados, evitando la dispersión de las mismas entre los distintos archivos y empresas de custodia, es una mayor garantía de interoperabilidad.
Pero estos efectos positivos únicamente tendrán lugar si el servicio de conservación de firmas y sellos electrónicos se implementa, tanto en sus aspectos legales como técnicos, de forma que no suponga una barrera para la creación de los archivos electrónicos y para la asunción de estos por los profesionales y órganos que vienen realizando esta función con los documentos en soporte papel. Ello supone que deberían seguir siendo estos quienes custodien y conserven íntegramente los documentos electrónicos auténticos, facilitándoles esta tarea mediante la externalización a los proveedores cualificados de operaciones concretas y, especialmente, del resellado de las firmas longevas.

\section{Agradecimientos}

Este trabajo ha sido financiado por la Cátedra Logisman de la Universidad de Zaragoza para la Gestión Tecnológica Documental.

\section{Referencias}

Bercovitz Rodríguez-Cano, Rodrigo (coord.) (2011). Manual de Derecho Civil (contratos). Madrid: Bercal.

Broseta Pont, Manuel; Martinez Sanz, Fernando (2010). Manual de Derecho Mercantil II. Madrid: Tecnos.

Dumortier, Jos (2016). Regulation (EU) No 910/2014 on Electronic Identification and Trust Services for Electronic Transactions in the Internal Market (eIDAS Regulation). https://ssrn.com/abstract=2855484

Jerez Delgado, Carmen (2015). Principios, definiciones y reglas de un derecho civil europeo: el Marco Común de Referencia (DCFR). Madrid: Boletín Oficial del Estado.

Lopez-Monis Gallego, Mónica (2003). Ámbito de aplicación de la nueva Ley de Servicios de la Información y de Comercio Electrónico. // Mateu de Ros, Rafael; LopezMonis Gallego, Mónica (coord.), Derecho de Internet, La Ley de Servicios de la Sociedad de la Información. Cizur Menor: Aranzadi.

Ministerio de Energía, Turismo y Agenda Digital (2016). Guía de notificación de servicios electrónicos de confianza cualificados y contenidos mínimos del informe de evaluación de la conformidad en el marco del Reglamento eIDAS. Madrid: Ministerio.

Muñoz Soro, José Félix; Nogueras-Iso, Javier (2014). La digitalización de documentos en la Administración de Justicia. // Ibersid. 8, 49-53.

Sirvent García, Jorge (2015). El depósito como contrato de servicios: Notas para su reforma a la luz del marco común de referencia. // Revista Aranzadi de derecho patrimonial. 37, 27-63

Soler Matutes, Pere (2016). El contrato de depósito de código fuente. // Soler Matutes, Pere et. al. (eds.), Manual de gestión y contratación informática. Cizur Menor: Aranzadi.

Uria, Rodrigo; Menéndez, Aurelio; Cortes, Luis Javier (2001). El contrato de depósito. // Uria, Rodrigo; Menéndez, Aurelio (eds.). Curso de Derecho Mercantil II. Madrid: Civitas.

Enviado: 2017-03-30. Segunda versión: 2017-09-06. Aceptado: 2017-09-06. 
